\title{
Evaluation of AD472, a live attenuated recombinant herpes simplex virus type 2 vaccine in guinea pigs
}

\author{
Mark N. Pricharda, ${ }^{a}$, Ravi Kaiwar ${ }^{a}, 2$, Winthrop T. Jackman ${ }^{a}$, Debra C. Quenelle ${ }^{b}$, Deborah J. \\ Collins $^{b}$, Earl R. Kern ${ }^{b}$, George M. Kemble ${ }^{a}$, and Richard R. Spaete ${ }^{a}{ }^{*}$ \\ aMedlmmune Vaccines Inc., 297 N Bernardo Avenue, Mountain View, CA 94043, USA \\ bUniversity of Alabama School of Medicine, 1600 6th Avenue S, Birmingham, AL 35233, USA
}

\section{Abstract}

An attenuated recombinant herpes simplex virus type 2 (HSV-2), designated as AD472, was constructed by deleting both copies of the $\gamma_{1} 34.5$ gene, UL55-56, UL43.5, and the US10-12 region from HSV-2 strain G. This virus was engineered to be a safe and effective live attenuated HSV-2 vaccine and was tested in the guinea pig model of genital herpes to evaluate its ability to protect from disease upon challenge with the wild type (wt) virus, HSV-2 (G). AD472 administered intramuscularly did not prevent infection or virus replication in the vaginal tract, but did reduce both lesion development and severity in a dose-dependent manner in guinea pigs challenged with the $w t$ virus. Frequency of reactivation from latency was low compared with that of the parent virus, HSV-2 (G). Immunization with AD472 at doses of $1 \times 10^{5} \mathrm{PFU}$ generally precluded colonization of the ganglia or establishment of latency by the challenge virus. Results presented here support the concept of a rationally engineered live attenuated vaccine for the prevention of the genital disease associated with infection by HSV-2.

\section{Keywords}

HSV-2; Attenuated vaccine; Guinea pig; Herpesvirus vaccine; Genital herpes

\section{Introduction}

The incidence of HSV-2 infections has increased continuously despite two decades of heightened public awareness of sexually transmitted diseases and the availability of effective antiviral treatments for HSV infections [10]. In untreated individuals, transmission is associated with recurrent infection and can occur in the presence or absence of symptoms and is one of the many contributing factors responsible for the spread of this disease in the population [25]. Prophylaxis with antiviral drugs, such as valacyclovir and famciclovir are effective in reducing the recurrence of genital herpes infections [19]; however, virus shedding and transmission to sexual partners can still occur during therapy, albeit at a reduced rate [8]. Although these drugs are useful in the treatment of primary disease and prevention of recurrent infections [25], they are not well suited to reduce the incidence of severe neonatal herpes infections that occur in approximately 1500 infants per year [14]. Precautionary cesarean section has been shown to reduce the transmission of the virus to neonates [2], but it is estimated

\footnotetext{
(C) 2005 Published by Elsevier Ltd.

*Corresponding author. E-mail address: E-mail: spaeter@medimmune.com (R.R. Spaete).

1Present address: University of Alabama at Birmingham, Birmingham, AL, USA.

${ }^{2}$ Present address: Novacea Inc., 601 Gateway Blvd., Suite 800, South San Francisco, CA 94080, USA.
} 
that 1580 excess cesarean births are performed for every poor neonatal outcome and the procedure carries the risk of increased maternal mortality [14].

Vaccination should reduce viral replication during primary infection and could theoretically reduce the incidence and severity of primary and recurrent infections. Reduced viral load in vaccinated individuals may also reduce transmission as has been observed previously with antiviral drugs [8]. Indeed, studies reviewed recently by Stanberry et al. suggest that prior exposure to HSV-1 may provide some protection from acquisition of genital HSV-2 infection and limits the severity of the disease as compared to HSV-1 naive individuals [21]. A similar effect was observed in HSV-2 seropositive individuals in that reinfection by a second strain of HSV-2 is infrequent [7]. The fact that HSV-2 seropositive women are at reduced risk for transmitting this virus to their infants supports the notion that vaccination could provide protection against neonatal herpesvirus infections [2].

Several strategies for vaccination against HSV-2 infection are being developed or are in clinical trials, but none have yet been approved. Among the strategies currently under development are vaccination with DNA, peptides, engineered subunits, inactivated virus, replicationdefective viruses and replication-competent live attenuated viruses [15]. Vaccination with plasmids expressing $\mathrm{gD}$ and $\mathrm{gB}$ was previously shown to afford some protection from challenge with the $w t$ virus in the mouse model of HSV-2 infection [9]. However, most of the clinical experience with HSV-2 vaccines has been with recombinant subunit vaccines consisting of one or both of the major HSV-2 surface glycoproteins (gB2 and gD2) formulated with adjuvant to increase their immunogenicity. While the subunit vaccine regimens proved to be immunogenic and generated high-titered neutralizing antibodies, a clinical trial using this approach vaccine was unable to demonstrate protection against acquisition ofHSV-2 infection suggesting that a neutralizing antibody response is insufficient to prevent infection with this virus [6]. Recent results with an engineered subunit vaccine and novel adjuvant has met with some success in clinical trials and appeared to be protective against clinically apparent disease in seronegative women, but appeared to be ineffective in men [23].

The precise nature of immunological responses that could be protective in a primary infection are not well understood; however, a live attenuated vaccine closely mimics natural viral infection and should elicit a broad and robust immune response. Live viral vaccines offer distinct advantages over other vaccine strategies since they provide a biologically appropriate context to deliver viral antigens to the immune system to access innate immunity and to generate both a cellular and humoral immune response. Thus, the immunity induced by such a virus might afford the same protection against superinfection as that observed in seropositive individuals. This strategy proved to be successful in a closely related herpesvirus, varicella zoster virus, and a live attenuated vaccine is currently in widespread use [16].

Previous studies with a live attenuated HSV-1-based recombinant virus vaccine, R7020, demonstrated that it was safe and well tolerated in guinea pigs, but data from a subsequent clinical trial suggested that this vaccine virus was likely overattenuated [3,18]. A secondgeneration live attenuated vaccine, RAV 9395, was designed to delete a similar subset of viral genes without debilitating the replication of the virus to the degree observed with R7020, and also proved to be both safe and effective in the guinea pig model of genital herpes [20]. In this report, we describe a rationally designed live attenuated HSV-2 viral vaccine that is similar in concept to RAV 9395 that was described in a previous report and proved to be both safe and effective in preclinical studies [20].

RAV 9395 contained deletions of both copies of $\gamma_{1} 34.5$ and exhibited a phenotype of marked neuroattenuation. Previous reports have described the partial reversion of the neuroattenuated phenotype associated with $\gamma_{1} 34.5$ mutations through the inappropriate temporal expression of 
the US11 gene product $[4,11]$, and the deletion of this region in AD472 was predicted to improve the stability of the vaccine candidates. The new vaccine candidate described in this report, AD472, contains deletions in the $\gamma_{1} 34.5$ gene, UL55-56, UL43.5, and the US10-12 region and was engineered to add an additional level of safety by increasing the genetic and phenotypic stability of this virus. AD472 replicates to high titers in cell substrates suitable for manufacturing live vaccines, and is both genetically and phenotypically stable. In this report, we describe results in the guinea pig model of genital herpes. The fact that guinea pigs develop external genital lesions that can be scored according to their severity, and exhibit recurrent infections in addition to vaginal virus replication makes this an attractive model to assess the efficacy of vaccine candidates. This model is the standard for assessing efficacy of vaccines for genital herpes, notwithstanding the lack of immunological reagents to assess immune responses in this species. Parameters for determining efficacy included induction of neutralizing antibody, infection rates, virus replication in the vaginal tract, lesion development and severity, and virus replication in external genital lesions.

\section{Materials and methods}

\subsection{Construction of recombinant viruses}

AD472 was constructed from a set of six overlapping cosmid clones that were co-transfected in vero cells to produce infectious virus. Cosmids 9394.54 and 9394.67 were derived from RAV 9394, the $t k^{-}$predecessor of RAV 9395 and were used to reproduce the same $\gamma_{1} 34.5$ and UL55-56 deletions as described inRAV9395 [20]. Cosmid clone scpt43.5was used to delete UL43.5 via a $7.3 \mathrm{~kb}$ deletion and cosmid AD467 was used to delete the US10-12 region in the final virus. Remaining wild type ( $w t)$ viral sequences were supplied by cosmids GK5 and GK22. The virus progeny from the cotransfection were plaque-purified and three independent virus stocks, AD468, AD471 and AD472 were produced. Viral DNA was purified from each of these isolates and transfected into MRC- 5 cells to produce the stocks of virus used in these studies.

\subsection{Phenotypic stability}

The phenotypic stability of three independently derived isolates of the vaccine virus was examined by the serial passage in the central nervous system (CNS) of mice. For each isolate, a group of 104 -week-old male BALB/c mice were inoculated intracranially with approximately $1 \times 10^{5} \mathrm{PFU}$ of the vaccine virus. At $72 \mathrm{~h}$ post-infection, the animals were euthanized and their brains excised and homogenized. Virus present in pooled brain homogenates was amplified in vero cells, and used to infect the next set of animals. A total of nine serial passages were performed, and the $\mathrm{LD}_{50}$ of the virus stocks was determined at passage 1,6, and 9 in 4-week-old male BALB/c mice. A standard $t$-test was used to determine the significance of the $\mathrm{LD}_{50}$ data and $p$-values of $\leq 0.05$ was considered to be statistically significant.

\subsection{Guinea pig pathogenicity studies}

Three groups of 15 Hartley guinea pigs (Charles River Labs) were inoculated intravaginally with swabs immersed in three concentrations of AD472 to evaluate the pathogenicity of the vaccine candidate. Two groups of 15 guinea pigs were inoculated intravaginally with two concentrations of HSV-2 (G) for comparison. Animals were observed daily for signs of systemic viral disease, lesions were scored for severity $[12,22]$, and vaginal samples were taken on days $1,3,5,7$, and 10 to quantify viral replication. External genital lesions were swabbed on days 5, 6, 7, 8, 9, and 10 . All swabs were placed in tubes containing $2.0 \mathrm{ml}$ of media, vortexed, and frozen at $-70{ }^{\circ} \mathrm{C}$ until titrated for $\mathrm{HSV}$. When all the samples were collected, they were thawed, diluted serially and viral titers were determined in primary rabbit kidney cells [13] using a microtiter cytopathic effect assay. The presence or absence of lesions and 
severity of lesions was assessed and recorded daily on Days 1-21 post-inoculation. Dorsal lumbosacral ganglia samples from animals infected with the vaccine virus were collected from two animals per group on Day 5 post-inoculation and three animals per group on Day 10 postinoculation to determine the presence of infectious virus in the ganglia. Significant mortality was observed in animals infected with HSV-2 (G), so ganglia were harvested from one animal per group on Days 5 and 10. Half of the surviving guinea pigs were anesthetized for collection of blood via cardiac puncture for determination of neutralizing antibody titers on Day 28 postinoculation. The remaining animals were observed for recurrent lesions through Day 56.

Guinea pigs were then anesthetized for a final blood collection and euthanasia.

\subsection{Challenge studies}

Six groups of 15 guinea pigs were inoculated intramuscularly with either cell culture media, or AD472 at $1 \times 10^{3}$ or $1 \times 10^{5} \mathrm{PFU}$. Twenty-two days post-immunization, guinea pigs were anesthetized for collection of blood samples via cardiac puncture. Animals were challenged with either $1 \times 10^{6}$ or $5 \times 10^{4} \mathrm{PFU} / \mathrm{ml} \mathrm{HSV}-2(\mathrm{G})$ on Day 28 by the intravaginal route. Each animal was examined daily, lesions were scored for severity and samples were taken to assess the replication of the challenge virus. Dorsal lumbosacral ganglia samples were collected from five infected animals per group on Days 34-38 post-inoculation to determine the presence of virus in the ganglia by co-cultivation on primary rabbit kidney fibroblasts. Terminal blood collections were performed via cardiac puncture prior to euthanasia and retrieval of ganglia. All remaining guinea pigs were monitored for lesions, and vaginal swabs were collected twice weekly until the end of the study (Day 60 post-viral inoculation). At euthanasia, a final blood sample was obtained from each animal for neutralizing antibody determination.

\subsection{Determination of neutralizing antibody titers}

To determine antibody levels, animals were bled under anesthesia (ketamine-xylazine) via a cardiac puncture on Days 28 and 56, post-viral inoculation. Blood was allowed to clot, centrifuged, and the serum frozen at $-20^{\circ} \mathrm{C}$. Neutralizing antibody titers were determined by a plaque reduction assay in six-well plates. Briefly, 40-60 PFU of HSV-2 (G) were placed in a tube, dilutions of sera were added without the addition of exogenous complement, and the final mixture was incubated for $60 \mathrm{~min}$ at $37^{\circ} \mathrm{C}$. The mixtures were then assayed for remaining infectious virus in duplicate on monolayers of primary rabbit kidney cells and 50\% endpoint neutralizing titers were calculated as the geometric mean of the groups as well as the standard deviation.

\subsection{Statistical evaluation}

To determine replication characteristics of the vaccine virus, animals given AD472 were compared to the group of HSV-2 (G)-infected guinea pigs. In the efficacy study, infection rates, peak virus titers, virus titer-day areas under the curves (AUC), peak lesion scores and lesion score-day AUC between vaccinated and unvaccinated animals were compared using the Mann-Whitney $U$-Rank Sum Test and $p$-values of 0.05 or less were considered to be statistically significant.

\section{Results}

\subsection{Construction of recombinant viruses and their replication characteristics}

AD472 is similar in many respects to RAV 9395; however, additional mutations were engineered into the genome that were specifically designed to improve the safety profile of this vaccine candidate by increasing its phenotypic stability. This virus was constructed from a set of six overlapping cosmid clones and its predicted genomic structure (Fig. 1) was confirmed by restriction fragment analysis of viral DNA and the virus appeared to be stable 
following five serial passages in cell culture (data not shown). Virion DNAs purified from each of these master virus stocks were transfected into MRC-5 cells and amplified in these cells to produce the working virus stocks used in studies presented here. AD472 replicated well in primary human fibroblast cells and achieved titers deemed suitable for manufacture in this cell substrate. This virus yielded titers of approximately $10^{6} \mathrm{PFU} / \mathrm{ml}$, which represented a 10 -fold reduction in replication efficiency than that observed previously with RAV 9395.

\subsection{Phenotypic stability}

To confirm that these engineered deletions conferred the desired phenotypic stability to the recombinant viruses, three separate isolates were serially passaged nine times through the CNS of mice and $\mathrm{LD}_{50}$ values were ascertained following passage 1, 6, and 9 (Fig. 2). Each of the independent isolates examined remained highly attenuated in the CNS even after nine serial passages and the $\mathrm{LD}_{50}$ for each isolate was not statistically different from unpassaged virus. These results suggest that this specific set of engineered mutations conferred both attenuation as well as phenotypic stability on each of the AD-viruses and AD472 was chosen for further evaluation.

\subsection{Replication, attenuation, and immunogenicity in the guinea pig model}

The guinea pig model of genital HSV-2 infection was used to confirm the attenuated nature of the vaccine candidate and to establish the replication characteristics in an established model of HSV-2 infection [12,22]. Animals inoculated by means of Dacron swabs immersed in a solution containing $5 \times 10^{5} \mathrm{PFU} / \mathrm{ml}$ of AD472 did not develop genital lesions and shed very low levels of virus, predominantly during the first few days after inoculation (Fig. 3). In contrast, animals inoculated with $5 \times 10^{5} \mathrm{PFU} / \mathrm{ml}$ of HSV-2 (G), all developed lesions and high titers of virus were shed for at least a week following infection. In the guinea pig model, primary lesions typically resolve by Day 21 ; however, lesions resulting from inoculation with this high dose of HSV-2 (G) were more severe and persisted in many animals at this point in time. Nevertheless, recurrent lesions were observed in the subset of animals that had resolved the primary infection with the $w t$ virus (data not shown). Essentially, no lesions were observed in animals inoculated with AD472 throughout the study and no recurrences were observed in any animals vaccinated with this virus. The large differences in lesion severity and virus replication between animals inoculated with AD472 or HSV-2 (G) confirmed that the vaccine virus is highly attenuated in this model and does not appear to cause any adverse reactions.

Dorsal root ganglia were obtained from animals in each group at 5 and 10 days post-infection to assess the ability of the viruses to establish an acute infection of the ganglia innervating the infected tissue. No virus was detected in the ganglia of the 15 animals infected with AD472, whereas ganglia from two of the four animals infected with $w t$ virus yielded infectious virus (data not shown). Thus, AD472 does not appear to replicate well enough in the vaginal tract to initiate an infection of the peripheral neurons with spread to the ganglia and has a greatly reduced capacity to replicate or establish latency in these ganglia, which is similar to results reported previously for RAV 9395 [20].

Immunogenicity of AD472 in this model was assayed by measuring neutralizing antibody titers from animals infected intravaginally with either AD472 or wt virus. Infection with either $5 \times$ $10^{5}$ or $1 \times 10^{6} \mathrm{PFU} / \mathrm{ml} \mathrm{HSV}-2$ (G) elicited a strong neutralizing antibody response by 28 days post-infection and titers exceeded $3.4\left(\log _{10}\right)$ by day 56 of the infection (Table 1). Animals inoculated with either $5 \times 10^{3}$ or $5 \times 10^{5} \mathrm{PFU} / \mathrm{ml}$ of AD472 developed detectable neutralizing titers by 28 days after infection and generated $\log _{10}$ titers of only 1.1 and 2.0, respectively, by Day 56 of the infection. A lower inoculum of $5 \times 10^{3} \mathrm{PFU} / \mathrm{ml}$ did not typically induce detectable neutralizing titers. In general, the titers elicited by AD472 were considerably lower than that 
induced by infection with the $w t$ virus, which was expected given the limited replication observed in this model.

\subsection{Protection from challenge with wt virus}

The effectiveness of this vaccine was evaluated in a challenge experiment with lesion development and virus replication as primary endpoints. In the protection study, two challenge doses were used; either a lower dose $\left(5 \times 10^{4} \mathrm{PFU} / \mathrm{ml}\right)$ or a higher dose $\left(1 \times 10^{6} \mathrm{PFU} / \mathrm{ml}\right)$ were used to challenge animals that received intramuscular injection with $1 \times 10^{3}$ or $1 \times 10^{5} \mathrm{PFU}$ of AD472 28 days prior to challenge. Animals immunized intramuscularly with $1 \times 10^{5} \mathrm{PFU}$ of AD472 developed neutralizing antibodies by 28 days after infection, and titers were indistinguishable from those elicited by intravaginal inoculation with the same quantity of this virus and evaluated at this same interval (Table 1). Thus, for AD472, both routes of administration were sufficient to induce the production of neutralizing antibodies and suggest that the animals had developed some humoral immunity prior to challenge.

Despite measurable neutralizing antibody titers, none of the groups had significant reductions in the virus titer-day AUC or the mean peak virus titer (Table 2). Immunized animals were, however, protected from disease. Guinea pigs that were immunized with either $1 \times 10^{5}$ or $1 \times$ $10^{3} \mathrm{PFU}$ of AD472 and challenged with $5 \times 10^{4} \mathrm{PFU} / \mathrm{ml}$ of $w t$ virus had a significant reduction in the lesion-day area under the curve (AUC) $(p<0.001)$, as well as mean peak lesion scores $(p<0.01)$ (Table 3). At the higher challenge dose of $1 \times 10^{6} \mathrm{PFU} / \mathrm{ml}$, vaccination with $1 \times$ $10^{5} \mathrm{PFU}$ of the vaccine virus also significantly reduced the lesion-day AUC $(p<0.001)$ and mean peak lesion scores $(p<0.05)$. However, vaccination with only $1 \times 10^{3} \mathrm{PFU}$ of AD472 was not effective against the high-challenge dose.

Recurrence rates were low in all groups (data not shown). The control groups, which received HSV-2 (G) without immunization, had rates of 11.1 and $0 \%$ for the $5 \times 10^{4}$ and $1 \times 10^{6} \mathrm{PFU} /$ $\mathrm{ml}$ challenge groups, respectively. Recurrence rates for animals receiving the higher challenge dose of $1 \times 10^{6} \mathrm{PFU} / \mathrm{ml}$ and immunized with $1 \times 10^{3}$ and $1 \times 10^{5} \mathrm{PFU}$ AD472 were 18 and $15 \%$, respectively, while animals receiving the lower challenge dose of $5 \times 10^{4} \mathrm{PFU} / \mathrm{ml}$ had recurrence rates of 7.1 and $6.6 \%$, respectively. Recurrent infections in all animals were minor in severity and no statistically significant differences were observed among the groups (data not shown).

Latent virus was recovered from 60 and $80 \%$ of unvaccinated animals challenged with HSV-2 (G) at the high-and low-challenge doses, respectively (Table 4). No latent virus was detected in any of the animals vaccinated with $1 \times 10^{5} \mathrm{PFU}$ of AD472 at the lower challenge dose and represented a significant reduction compared to unvaccinated controls $(p<0.05)$. There were no significant reductions in latent virus recovery in other groups of vaccinated animals.

\section{Discussion}

The purpose of these studies was to describe the rationale and construction of a live attenuated vaccine candidate, AD472 that was designed specifically as a vaccine for HSV-2 and to evaluate its safety, immunogenicity, and efficacy in an animal model of genital herpes. The genetic characteristics of this virus are similar to that described previously for RAV 9395 [20], but carries additional genetic modifications designed to improve the safety profile of the virus by reducing the potential for phenotypic reversion. The deletion of $\gamma_{1} 34.5$ in both these viruses is perhaps the most important mutation that contributes to its safety profile.

Recombinant viruses that are unable to express ICP34.5 are incapable of replicating in the CNS of mice following direct cerebral inoculation [5,24]. Subsequent studies demonstrated that the phenotype of low neurovirulence in other $\gamma_{1} 34.5$ deletion viruses could be reverted through altered expression kinetics of the US11 gene [4]. Thus, the deletion of both US11 as well as 
$\gamma_{1} 34.5$ would be predicted to possess a low reversion rate by eliminating this mechanism of reversion and experiments presented here appear to support that hypothesis. The constellation of mutations in AD472 clearly attenuates the replication of the virus in both the mouse and guinea pig. The low neurovirulence phenotype also appears to be stable even upon serial passage through the CNS of susceptible mice. The capacity to produce low neurovirulence and maintain genetic stability are essential characteristics for any live attenuated HSV-2 vaccine and represent a significant improvement over previous generations of live attenuated HSV vaccine candidates.

The intravaginal inoculation of young guinea pigs with HSV-2 results in a predictable course of primary disease characterized by replication in the genital tract followed by the development of external vesicular lesions that proceed to lesions or ulcers. Animals typically shed high titers of virus during the initial few days of infection, which wane and resolve within the first week to 10 days. External genital lesions appear approximately 4 days following inoculation and peak with a varying level of severity around day 10 and resolve after 18-20 days post-infection. Recurrent disease can also occur in animals following the resolution of the primary infection and is characterized by the appearance of mild external vesicular genital lesions that resolve in 1-2 days, but can later recur [12,22]. Animals inoculated with AD472 did not follow this typical course of infection. Infection with $5 \times 10^{5} \mathrm{PFU} / \mathrm{ml}$ of this virus resulted in little or no virus replication in the vaginal tract and essentially no lesions resulted from the infection. This level of attenuation is similar to that described previously with both R7020 as well as RAV 9395 and suggests that this virus would be highly attenuated in humans as well $[18,20]$. The potential for AD472 to establish a latent infection also appears to be greatly reduced since the replication of this virus in the vaginal tract was so low that no virus was detected in any ganglia from animals infected with this virus at Day 5 of the infection, whereas virus was isolated from ganglia obtained from both animals infected with HSV-2 (G) at the same period of time. Previous results with RAV 9395 suggested that the establishment of latent infections was inefficient compared to $w t$ virus and is consistent with the results with AD472.

The challenge studies presented here used two different concentrations of AD472 and two different challenge doses to help determine the degree of protection afforded by different doses of the vaccine virus. Intramuscular injection of $1 \times 10^{3} \mathrm{PFU}$ of the vaccine virus did not elicit a measurable neutralizing antibody response in most of the immunized animals by 28 days after infection; however, the vaccine was still capable of protecting animals from disease at the lower challenge dose of $5 \times 10^{4} \mathrm{PFU} / \mathrm{ml}$ as measured by the statistically significant reduction in both the mean peak lesion score as well as the lesion-day AUC (Table 3). When the challenge dose was increased by 20 -fold $\left(1 \times 10^{6} \mathrm{PFU} / \mathrm{ml}\right)$, no significant protection was observed. Intramuscular injection of $10^{5} \mathrm{PFU}$ of AD472 improved the efficacy of the vaccine and statistically significant protection was observed at both the low- and high-challenge doses of $w t$ virus. The induction of measurable neutralizing antibody titers clearly is not required to confer protection in this model and cellular-mediated immunity likely contributes to the protective immune response. However, the appearance of neutralizing antibody at the higher vaccine dose might be an indicator of a threshold level of vaccine replication required to establish a robust immune response that is protective even at high-challenge doses. The fact that the higher dose of vaccine could reduce the establishment of latency as observed in the animals receiving the low-challenge dose could also be interpreted as a result of a superior immune response.

AD472 is replication competent and presents a full complement of viral proteins to the immune system with the exception of the genes that were deleted in an attempt to confer the desired characteristics to its phenotype. This vaccine is immunogenic, phenotypically and genotypically stable and is capable of replicating to sufficiently high titers in primary human fibroblast cells (an approvable cell substrate) that indicates feasibility of manufacture. In 
general, live attenuated vaccines have the greatest potential to protect animals from infection and disease. This vaccine did protect guinea pigs from disease, yet was unable to protect them from infection. This effect was reported previously in a guinea pig challenge study for RAV 9395 and was also expected for AD472, since it is so closely related to RAV 9395 [20]. AD472 replicates well in vitro, but little replication could be documented in the guinea pig model and it is possible that the level of replication is insufficient to elicit mucosal immune response that is capable of protecting animals from subsequent infection. The additional mutations in UL55-56, UL43.5, and the US10-12 region that confer phenotypic stability may also be responsible for the poor replication in vivo. Increasing the dose of the vaccine might improve the poor replication characteristics of this virus in vivo and might result in a superior immune response. A different strategy using replication defective viruses has also been examined in an animal model similar to that described here $[1,17]$. These viruses are likely to be safer than live attenuated vaccines because they are incapable of producing infectious virus in the host. Vaccination with these defective viruses did not protect guinea pigs from infection, but did protect them from disease upon challenge with $w t$ virus, but required higher doses of the vaccine virus $\left(10^{6}-10^{7} \mathrm{PFU}\right)$ and two vaccinations were required to afford a high degree of protection. Although these vaccines are safer than live attenuated vaccines, they may not be capable of generating a robust and durable immune response and the high doses of virus required might make them difficult to manufacture. Subunit vaccines should be safer than either of these strategies but have met with mixed results in human clinical trials $[6,23]$. These vaccines typically generate neutralizing antibody titers, but this alone is likely insufficient to confer a high degree of protection [6]. Indeed, results presented here argue that a neutralizing antibody response is not required to protect animals from disease and cellular-mediated immunity clearly plays an important role. The long-term effects of the adjuvants required to produce the desired immune responses with the subunit approach are unknown and represent a significant hurdle in the development of this strategy.

Live attenuated viral vaccines have proven to generate protective and durable immunity to many virus diseases. The presentation of viral antigens in the right biological context might be important for the generation of an appropriate immune response. The results presented here suggest that the development of a live attenuated vaccine for HSV-2, such as AD472, is an effective strategy and warrants further investigation.

\section{Acknowledgements}

The authors thank Bridgett Herrod and Kourtney Gustin for expert technical assistance in the animal studies and the neutralizing antibody experiments. This work was supported by SBIR grant AI43139-02A1 to MedImmune Vaccines from the NIAID, NIH.

\section{References}

1. Boursnell ME, Entwisle C, Blakeley D, Roberts C, Duncan IA, Chisholm SE, et al. A genetically inactivated herpes simplex virus type 2 (HSV-2) vaccine provides effective protection against primary and recurrent HSV-2 disease. J Infect Dis 1997;175:16-25. [PubMed: 8985191]

2. Brown ZA, Wald A, Morrow RA, Selke S, Zeh J, Corey L. Effect of serologic status and cesarean delivery on transmission rates of herpes simplex virus from mother to infant. JAMA 2003;289:203209. [PubMed: 12517231]

3. Cadoz, M.; Micoud, M.; Seigneurin, J., et al. Presented at the program and abstracts for the 32nd interscience conference on antimicrobial agents and chemotherapy; Anaheim, CA. 1992.

4. Cassady KA, Gross M, Roizman B. The second-site mutation in the herpes simplex virus recombinants lacking the $\gamma_{1} 34.5$ genes precludes shutoff of protein synthesis by blocking the phosphorylation of eIF-2alpha. J Virol 1998;72:7005-7011. [PubMed: 9696792]

5. Chou J, Kern ER, Whitley RJ, Roizman B. Mapping of herpes simplex virus-1 neurovirulence to gamma 134.5, a gene nonessential for growth in culture. Science 1990;250:1262-1266. [PubMed: 2173860] 
6. Corey L, Langenberg AG, Ashley R, Sekulovich RE, Izu AE, Douglas JM Jr, et al. Chiron HSV Vaccine Study Group. Recombinant glycoprotein vaccine for the prevention of genital HSV-2 infection: two randomized controlled trials. JAMA 1999;282:331-340. [PubMed: 10432030]

7. Corey L, Spear PG. Infections with herpes simplex viruses (1). N Engl J Med 1986;314:686-691. [PubMed: 3005858]

8. Corey L, Wald A, Patel R, Sacks SL, Tyring SK, Warren T, et al. Once-daily valacyclovir to reduce the risk of transmission of genital herpes. N Engl J Med 2004;350:11-20. [PubMed: 14702423]

9. Domingo C, Gadea I, Pardeiro M, Castilla C, Fernandez S, Fernandez-Clua MA, et al. Immunological properties of a DNA plasmid encoding a chimeric protein of herpes simplex virus type 2 glycoprotein B and glycoprotein D. Vaccine 2003;21:3565-3574. [PubMed: 12922084]

10. Fleming DT, McQuillan GM, Johnson RE, Nahmias AJ, Aral SO, Lee FK, et al. Herpes simplex virus type 2 in the United States, 1976 to 1994. N Engl J Med 1997;337:1105-1111. [PubMed: 9329932]

11. He B, Chou J, Brandimarti R, Mohr I, Gluzman Y, Roizman B. Suppression of the phenotype of $\gamma_{1} 34$.5-herpes simplex virus 1: failure of activated RNA-dependent protein kinase to shut off protein synthesis is associated with a deletion in the domain of the alpha47 gene. J Virol 1997;71:60496054. [PubMed: 9223497]

12. Kern ER. Acyclovir treatment of experimental genital herpes simplex virus infections. Am J Med 1982;73:100-108. [PubMed: 6285699]

13. Kern ER, Overall JC Jr, Glasgow LA. Herpesvirus hominis infection in newborn mice. I. An experimental model and therapy with iododeoxyuridine. J Infect Dis 1973;128:290-299. [PubMed: 4353930]

14. Kimberlin DW. Neonatal herpes simplex infection. Clin Microbiol Rev 2004;17:1-13. [PubMed: 14726453]

15. Koelle DM, Corey L. Recent progress in herpes simplex virus immunobiology and vaccine research. Clin Microbiol Rev 2003;16:96-113. [PubMed: 12525427]

16. Kuter B, Matthews H, Shinefield H, Black S, Dennehy P, Watson B, et al. Ten year follow-up of healthy children who received one or two injections of varicella vaccine. Pediatr Infect Dis $\mathbf{J}$ 2004;23:132-137. [PubMed: 14872179]

17. McLean CS, Erturk M, Jennings R, Challanain DN, Minson AC, Duncan I, et al. Protective vaccination against primary and recurrent disease caused by herpes simplex virus (HSV) type 2 using a genetically disabled HSV-1. J Infect Dis 1994;170:1100-1109. [PubMed: 7963701]

18. Meignier B, Longnecker R, Roizman B. In vivo behavior of genetically engineered herpes simplex viruses R7017 and R7020: construction and evaluation in rodents. J Infect Dis 1988;158:602-614. [PubMed: 2842408]

19. Reitano M, Tyring S, Lang W, Thoming C, Worm AM, Borelli S, et al. International Valaciclovir HSV Study Group. Valaciclovir for the suppression of recurrent genital herpes simplex virus infection: a large-scale dose range-finding study. J Infect Dis 1998;178:603-610. [PubMed: 9728526]

20. Spector FC, Kern ER, Palmer J, Kaiwar R, Cha TA, Brown P, et al. Evaluation of a live attenuated recombinant virus RAV 9395 as a herpes simplex virus type 2 vaccine in guinea pigs. J Infect Dis 1998;177:1143-1154. [PubMed: 9592996]

21. Stanberry LR, Cunningham AL, Mindel A, Scott LL, Spruance SL, Aoki FY, et al. Prospects for control of herpes simplex virus disease through immunization. Clin Infect Dis 2000;30:549-566. [PubMed: 10722443]

22. Stanberry LR, Kern ER, Richards JT, Abbott TM, Overall JC Jr. Genital herpes in guinea pigs: pathogenesis of the primary infection and description of recurrent disease. J Infect Dis 1982;146:397404. [PubMed: 6286797]

23. Stanberry LR, Spruance SL, Cunningham AL, Bernstein DI, Mindel A, Sacks S, et al. Glycoproteind-adjuvant vaccine to prevent genital herpes. N Engl J Med 2002;347:1652-1661. [PubMed: 12444179]

24. Whitley RJ, Kern ER, Chatterjee S, Chou J, Roizman B. Replication, establishment of latency, and induced reactivation of herpes simplex virus $\gamma_{1} 34.5$ deletion mutants in rodent models. J Clin Invest 1993;91:2837-2843. [PubMed: 8390490] 
25. Whitley RJ, Roizman B. Herpes simplex virus infections. Lancet 2001;357:1513-1518. [PubMed: 11377626] 


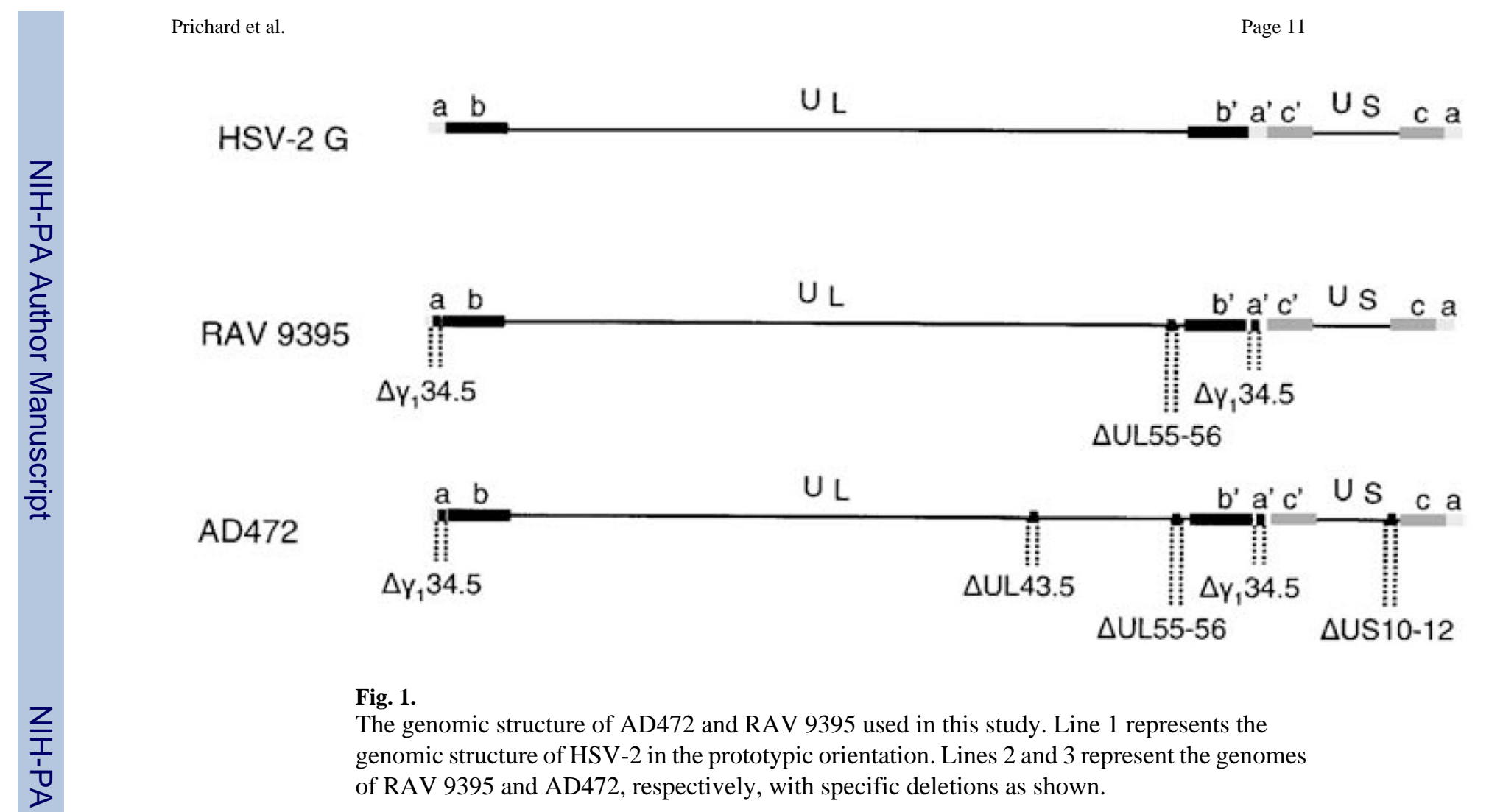




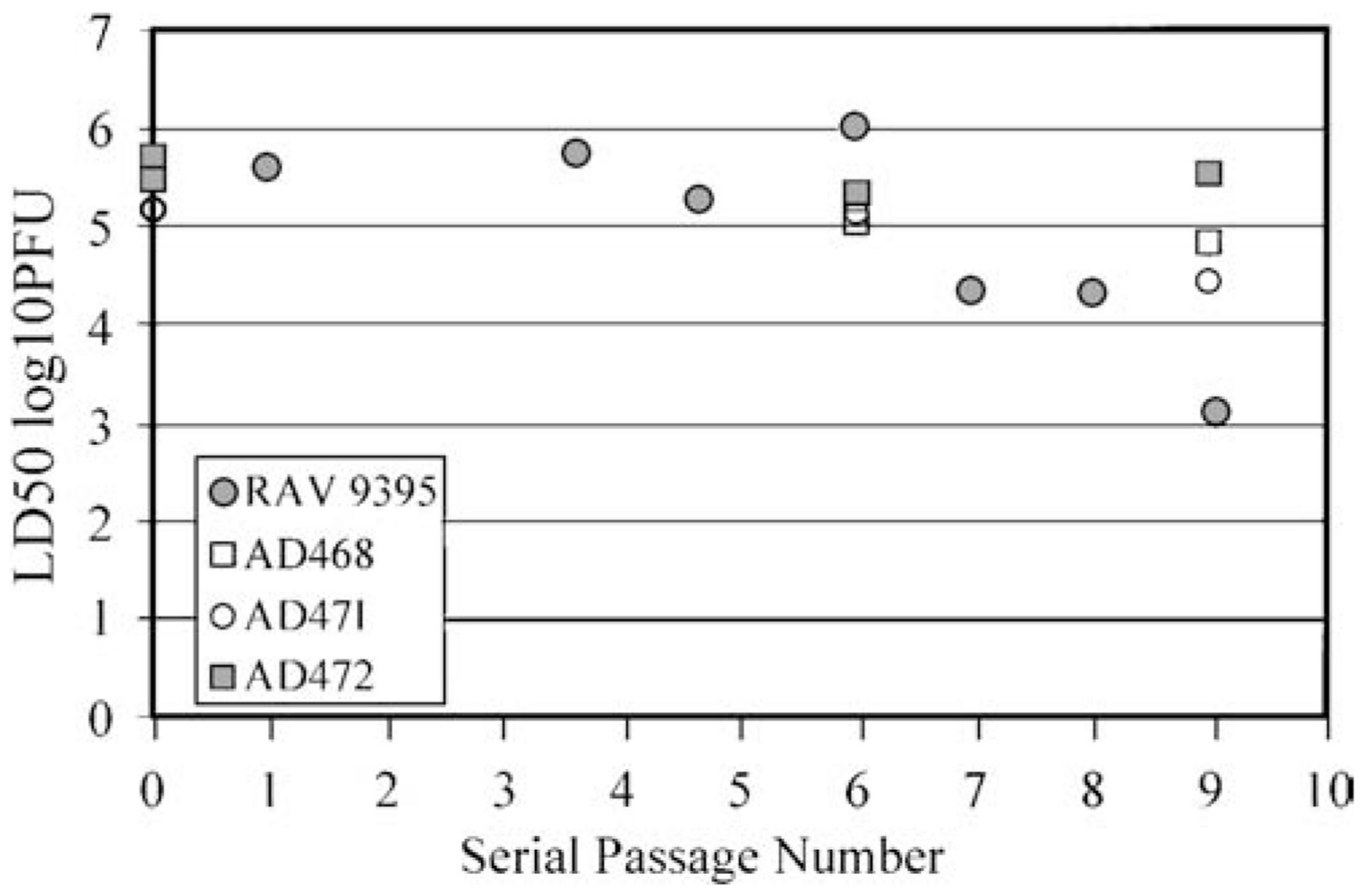

Fig. 2.

Relative neurovirulence of virus isolates upon serial passage in the CNS of mice. The $\mathrm{LD}_{50}$ of RAV9395, AD468, AD471, and AD472 is shown following the number of serial passages through the CNS of mice indicated on the bottom axis. 

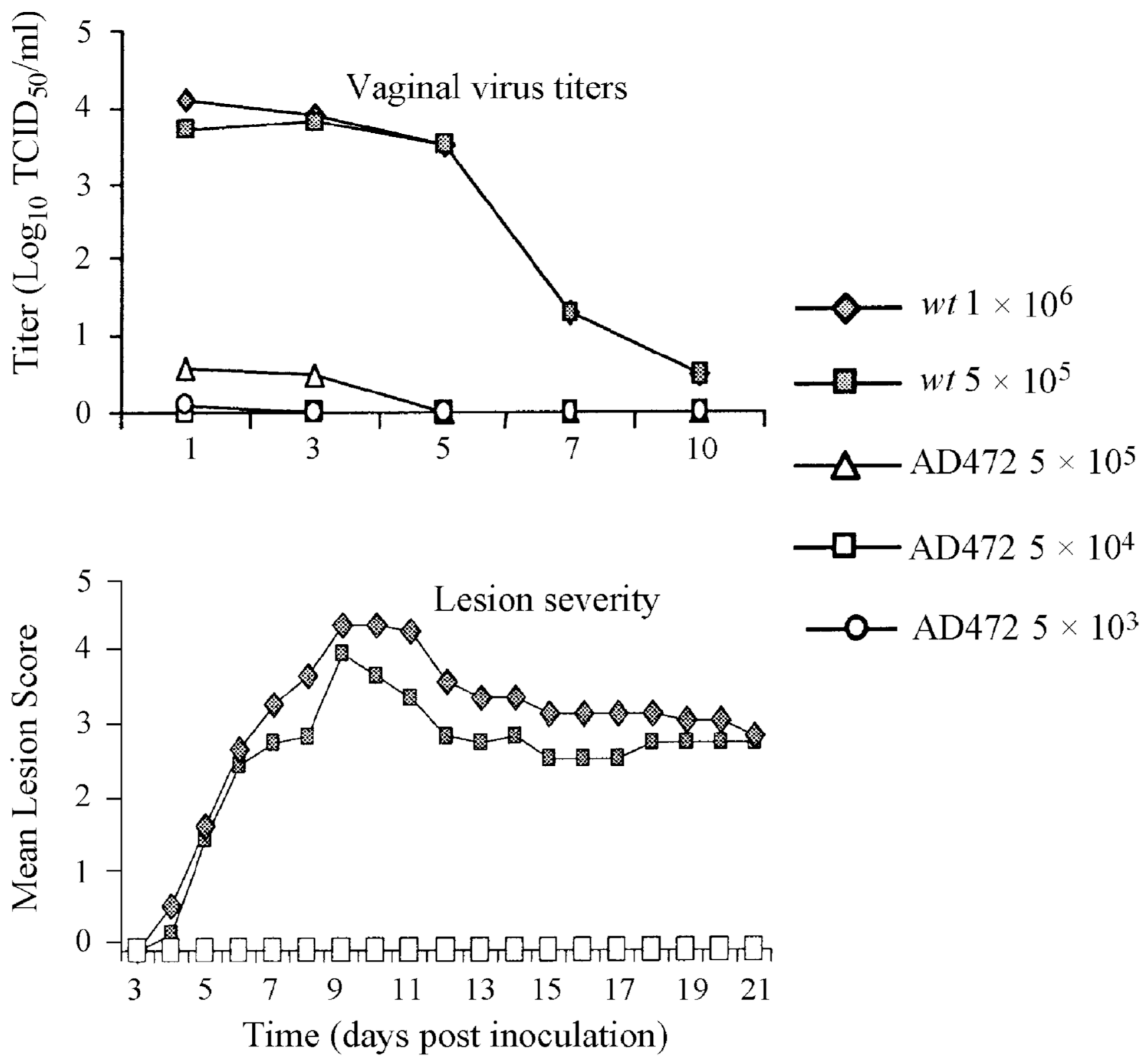

Fig. 3.

Pathogenesis of AD472 in guinea pigs following intravaginal inoculation. The top panel depicts the titers of shed virus for AD472 and $w t$ virus following inoculation with the titers shown. The bottom panel represents lesion severity for the same viruses over time. 


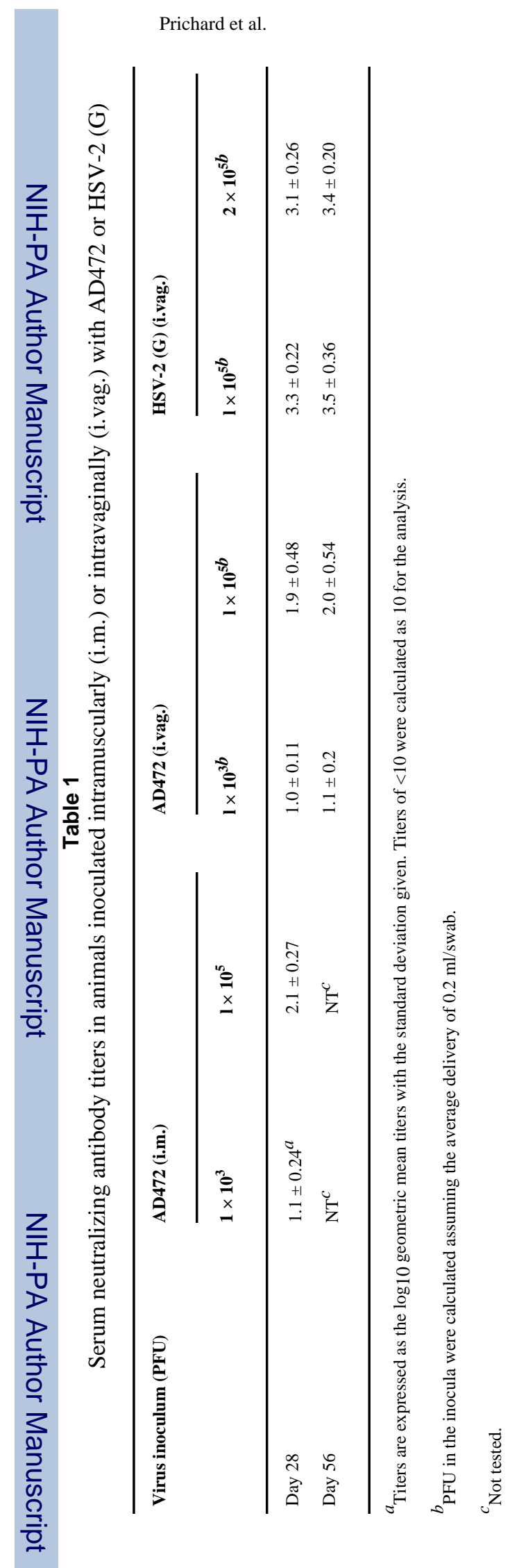

Page 14 


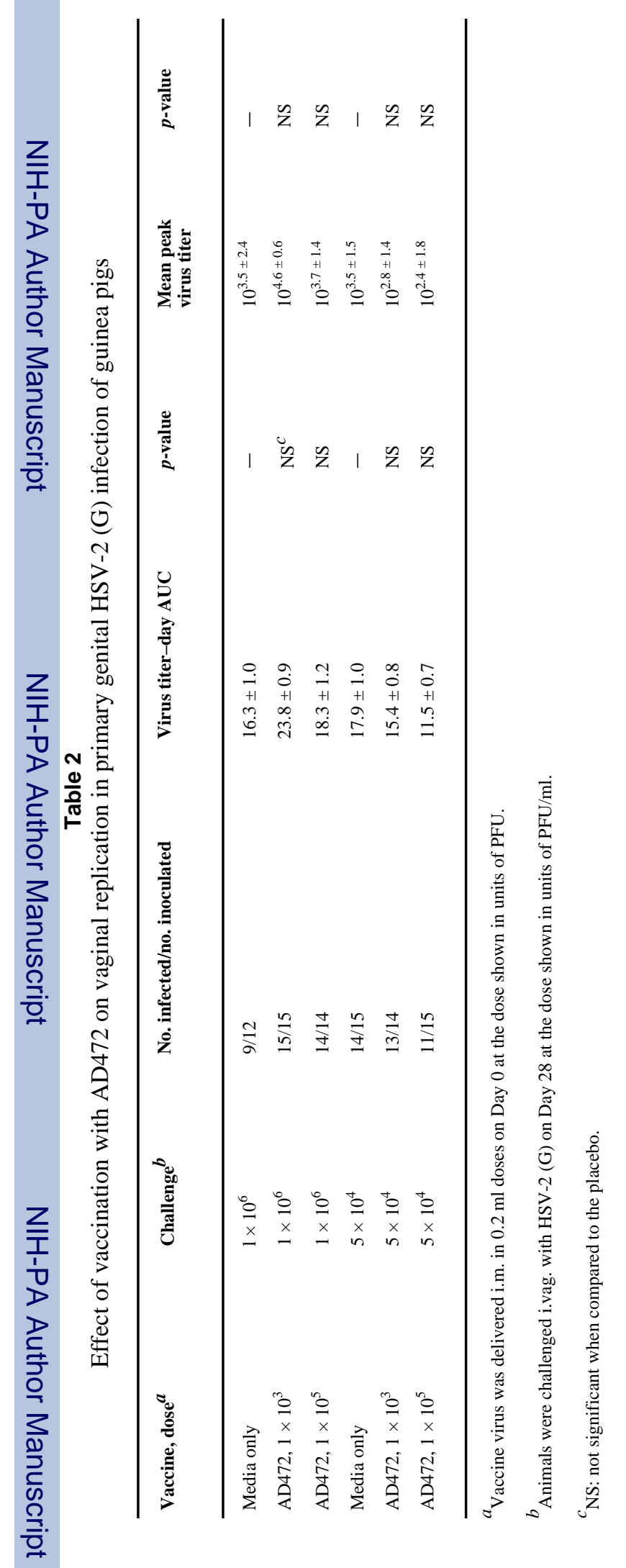


Prichard et al.

Page 16

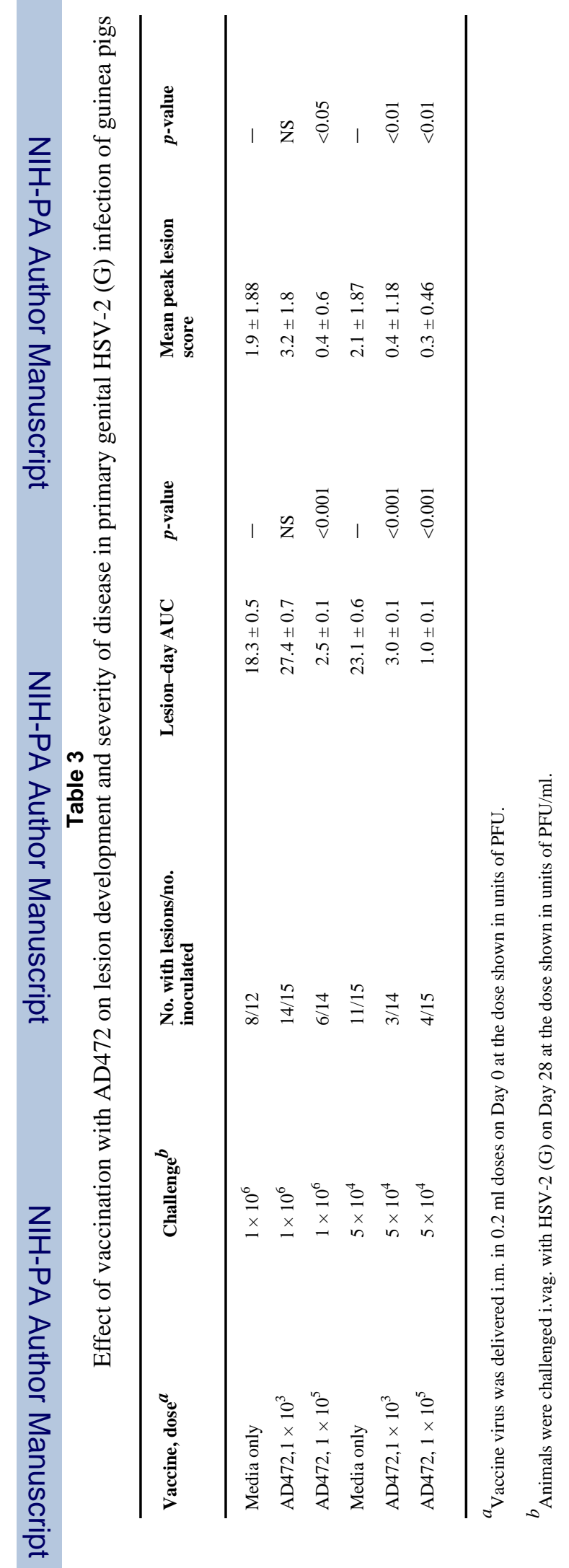

Vaccine. Author manuscript; available in PMC 2009 July 30. 
Table 4

Rates of reactivation from dorsal lumbosacral ganglia for vaccinated and unvaccinated guinea pigs challenged with HSV-2 (G)

\begin{tabular}{llll}
\hline Vaccine, dose $^{a}$ & Challenge $\boldsymbol{b}$ & No. positive/cultured & $\begin{array}{c}\text { Percent positive } \\
\text { Mean days post- } \\
\text { cocultivation to } \\
\text { CPE }\end{array}$ \\
\hline Media only & $1 \times 10^{6}$ & $3 / 5$ & 9.33 \\
AD472, $1 \times 10^{3}$ & $1 \times 10^{6}$ & $3 / 5$ & 9.67 \\
AD472, $1 \times 10^{5}$ & $1 \times 10^{6}$ & $1 / 5$ & 60 \\
Media only & $5 \times 10^{4}$ & $4 / 5$ & 10.00 \\
AD472, $1 \times 10^{3}$ & $5 \times 10^{4}$ & $1 / 5$ & 11.75 \\
AD472, $1 \times 10^{5}$ & $5 \times 10^{4}$ & $0 / 5^{c}$ & 9.00 \\
\hline
\end{tabular}

\footnotetext{
${ }^{a}$ Vaccine virus was delivered i.m. in $0.2 \mathrm{ml}$ doses on Day 0 at the dose shown in units of PFU.

${ }^{b}$ Animals were challenged i.vag. with HSV-2 (G) on Day 28 at the dose shown in units of PFU/ml.

${ }^{c}$ Significant $(p<0.05)$ when compared to the appropriate control.
} 